superior to an operation requiring a 2-day hospitalization from a socioeconomic standpoint.

Another advantage to this technique is that it can be performed as part of a complete echo-endoscopic mediastinal staging procedure (endobronchial ultrasound + endoscopic ultrasound), which can now allow for the evaluation and biopsy of all mediastinal lymph node stations during a single outpatient endoscopic procedure. Single-port thoracoscopy cannot provide complete mediastinal staging, therefore necessitating additional procedures in cases requiring it.

Moishe Liberman, MD, PhD CHUM Endoscopic Tracheobronchial and Oesophageal Center Division of Thoracic Surgery University of Montreal Montreal, Quebec, Canada

http://dx.doi.org/10.1016/ j.jtcvs.2012.12.019

\section{CARDIAC CYCLE EFFICIENCY DURING COUNTERPULSATION}

\section{To the Editor:}

We read with great interest the recent article by Onorati and colleagues, ${ }^{1}$ whose findings are in accordance with our own data obtained in an animal model arranged to investigate the effects of intra-aortic balloon pump (IABP)/heart volume ratio modification. ${ }^{2}$ We congratulate them for having addressed such a controversial topic and for shedding additional light on the IABP weaning method. Indeed, this publication underlines the superiority of progressive volume variation relative to the traditional rate reduction method as a procedure for IABP weaning, as we hypothesized on the basis of the negative effects of reducing the IABP/heart volume ratio, in terms of both hemodynamic performance and metabolic response.

Onorati and colleagues ${ }^{1}$ used the cardiac cycle efficiency (CCE), derived from the arterial pressure waveform, as their measure of hemodynamic performance. The CCE expresses the ability of the cardiovascular system to maintain homeostasis at different energetic levels resulting from simultaneous interactions among pump function (both mechanical and electrical contributions), the arterial system, venous return, and the pulmonary circulation. $^{3}$

We noticed, however, that patients in both groups had high CCEs. The value of the CCE is always less than 1 , because part of energy is lost during heart work and cannot be totally recovered (an increase in entropy). This "efficiency"- unlike the purely mechanical performance, which is always between 0 and $1-$ can also have in vivo negative values, and this represents a compensatory mechanism to activate the support for the body's compartments that are not working properly. ${ }^{3}$

Such high CCE values in the article of Onorati and colleagues ${ }^{1}$ might be attributable to underdamping or resonance artifacts that frequently affect blood pressure measurements in operating rooms and intensive care units and cause severe overestimation of systolic blood pressure and incorrect estimation of hemodynamic parameters when the pulse contour method is used. ${ }^{4}$ It is well known that patients undergoing cardiovascular surgery are at high risk of artifacts caused by underdamping because of high vascular stiffness, advanced age, and other conditions. ${ }^{4}$ In addition, the dynamic impedance is strongly influenced by the balloon in patients with IABPs, and as a result artifacts are very common, affecting the diastolic peak. In our opinion, great attention must therefore be paid to the accuracy of the pressure signals received during IABP weaning procedures.

It is also important to consider that the damping coefficients of standard transducer systems have been manufactured with the aim of obtaining the highest level of detail available from the arterial wave signal while maintaining the risk of underdamping as low as possible for the whole patient population, and not a specific group of patients. Extra damping must therefore be introduced to minimize the distorting effects of the measurement system's tendency to resonate. Nonetheless, the damping coefficient obtained is frequently insufficient, and resonance artifacts may affect the morphology and amplitude of the recorded pressure wave. Under these conditions, a dedicated transducer manufactured for limiting resonant effect is an useful device, although interpretation of the resulting waveform does require some experience. ${ }^{5}$

We would appreciate comments from Onorati and colleagues ${ }^{1}$ regarding whether this issue was addressed and how they proceeded to limit these underdamping artifacts.

We are grateful to Onorati and colleagues ${ }^{1}$ for sharing their experience and knowledge in this excellent study. An elucidation and comment of the point discussed here would be helpful for a better understanding of the nuances of IABP pathophysiology and management in these patients.

Sandro Gelsomino, $M D, P h D$ Salvatore Mario Romano, PhD Department of Heart and Vessels

Careggi Hospital Florence, Italy

\section{References}

1. Onorati F, Santini F, Amoncelli E, Campanella F, Chiominto B, Faggian G, et al. How should wean my next intra-aortic balloon pump? Differences between progressive volume weaning and rate weaning. J Thorac Cardiovasc Surg. Epub 2012 Apr 19

2. Gelsomino S, Renzulli A, Rubino AS, Romano SM, Lucà $F$, Valente $S$, et al. Effects of $1: 1,1: 2$ or 1:3 intra-aortic balloon counterpulsation/heart support on coronary haemodynamics and cardiac contractile efficiency in an animal model of myocardial ischaemia/reperfusion. Eur J Cardiothorac Surg. 2012;42:325-32

3. Romano SM. Cardiac cycle efficiency: a new parameter able to fully evaluate the dynamic interplay of the cardiovascular system. Int J Cardiol. 2012; 155:326-7.

4. Romagnoli S, Romano SM, Bevilacqua S, Lazzeri C, Gensini GF, Pratesi C, et al. Dynamic 
response of liquid-filled catheter systems for measurement of blood pressure: precision of measurements and reliability of the pressure recording analytical method with different disposable systems. J Crit Care. 2011;26:415-22.

5. Ragosta M. Textbook of clinical hemodynamics. Philadelphia: Saunders; 2008.

$$
\begin{array}{r}
\text { http://dx.doi.org/10.1016/ } \\
\text { j.jtcvs.2012.11.086 }
\end{array}
$$

\section{Reply to the Editor:}

We are grateful to Drs Gelsomino and Romano for their interest in our article. ${ }^{1}$ They raise several important issues related to the adequacy of investigating the cardiovascular system by means of the pressure recording analytical method (PRAM) and to the methods used to attenuate the risk of underdamping/resonance artifacts in vivo, potentially responsible for an incorrect measurement of hemodynamic parameters. ${ }^{1,2}$

Dynamic response artifacts are often observed in patients receiving peripheral monitoring based on pulse contour analysis. ${ }^{3}$ In particular, underdamping effects are frequently observed whenever the stiffness of the cardiovascular system, including the apparatus for pulse pressure "transduction," is augmented. 3,4 Therefore, conditions such as systemic arterial hypertension, severe calcified ascending aorta, diffuse atherosclerosis of the aortic arch, systemic vasoconstriction, hypovolemic conditions, and a stiff transduction system (related to the use of numerous stopcocks, stiff tubes, and arterial transduction catheters) all contribute to increase the risk for dynamic response artifacts. ${ }^{3,4}$

Although transducers ad hoc manufactured to avoid underdamping artifacts exist on the market, ${ }^{3}$ they were not available at our institution at the time of the study and therefore were not used. Accordingly, our detection system might have been unable to recognize and consequently "clean" possible dynamic artifacts.

However, as for any detection system based on pulse contour analysis, the recorded variables were validated by an expert operator. For a correct interpretation of any data, the PRAM method undeniably requires a proper recognition of the dicrotic notch (which can be easily misrecognized by the machine and in cases of underdamping/resonance artifacts). Therefore, back to Gelsomino and Romano's question, no data were collected in our study unless validated by a fully trained operator. Therefore whenever artifacts existed, the detecting system was "restarted" to achieve a correct recognition of the pulse wave, thus collecting data only when the system properly interpreted the pulse.

An additional troubleshooting maneuver was to move to a different arterial site to derive a "correct" pulse wave (mainly the common femoral arterial access, where artifacts were less commonly encountered in our experience). Furthermore, the transduction system was a priori optimized by using only 1 stopcock and flexible tubing/arterial catheters, and only $20 \mathrm{~F}$ catheters for radial transduction or $18 \mathrm{~F}$ catheters for femoral transduction.

These problems were rarely encountered during the study because of the setting and the peculiar hemodynamic pattern of the enrolled population: $\mathrm{Pa}-$ tients weaned from an intra-aortic balloon pump (IABP) are usually vasodilated (because of the IABP effect or the concomitant use of vasodilating drugs); moreover, patients with severe atherosclerotic aortic arch or thoracic descending aorta did not undergo transfemoral IABP because of institutional policies (and thus were not enrolled in this study). Given the peculiar design of the trial, based on the hemodynamic monitoring and surveillance of a potential perioperative low cardiac output state after weaning, all patients were maintained at proper preload (central venous pressure maintained at 8-12 $\mathrm{mm} \mathrm{Hg}$ ) and afterload states: thus, conditions such as vasoconstriction and hypovolemia, potentially favoring underdamping/resonance artifacts, were avoided. On the basis of this approach, it can be easily recognized that the most common causes of underdamping were prevented but overall rare in this specific patient population.

In addition, we want to underscore 3 major validation aspects of our study:

1. Before using PRAM, we performed a "cross-check" validation of data via a pilot study. We analyzed the reliability of hemodynamic indices derived from PRAM compared with those derived by the traditional SwanGanz thermodilution method. As in other reports, ${ }^{5}$ this preliminary study demonstrated a perfect agreement between PRAM and Swan-Ganz data, unless paroxysmal high-rate atrial fibrillation occurred. However, a new onset of high-rate atrial fibrillation did not occur in our patients during the weaning trial. Of note, a new onset of high-rate atrial fibrillation at our institution is considered a contraindication to the progression of an IABP-weaning trial.

2. Our trial was designed to investigate at 360 degrees the impact of the 2 weaning strategies on the entire cardiovascular system. Accordingly, hemodynamic and biochemical indices were collected, such as troponin I and lactate, the former showing the myocardial perfusion and the latter showing the adequacy of peripheral "oxygenation." Again, peripheral lactate confirmed the superiority of the "volumedeflation" method versus the "ratereduction" strategy in terms of peripheral perfusion.

3. Romagnoli and colleagues ${ }^{3}$ have demonstrated that whenever underdamping/resonance effects exist, significant differences are recorded between hemodynamic data derived from "conventional" PRAM and those derived from underdamping/ resonance-corrected PRAM by means of specific transducers. As we mentioned, these transducers were not available at the time we started the study. Furthermore, an 